\title{
Comparison of Median Sensory Conduction Values Proximal and Distal to Wrist Segment in Mild Carpal Tunnel Syndrome
}

\author{
SERPIL Demirci, MD ${ }^{1)}$, SerPIL SAVAŞ, MD²), Birkan Sonel, MD ${ }^{3)}$ \\ ${ }^{1)}$ Neurology Department, Faculty of Medicine, Süleyman Demirel University: Hosdere \\ Caddesi, 160/8, Cankaya, 06900, Ankara-Turkey. TEL +90 246-2326659/199 \\ ${ }^{2)}$ Physical Therapy and Rehabilitation Department, Faculty of Medicine, Süleyman Demirel \\ University \\ ${ }^{3)}$ Physical Therapy and Rehabilitation Department, Faculty of Medicine, Ankara University
}

\begin{abstract}
In carpal tunnel syndrome, depending on the severity and duration of compression, varied degrees of demyelination or axonal degeneration leads to low action potential amplitude with wrist stimulation. Amplitude drop across the lesion can distinguish demyelination from axonal degeneration. In 61 control and 127 consecutive CTS patient hands we recorded antidromic sensory nerve action potentials after palm and wrist stimulation to compare amplitude ratios. The latency, amplitude and velocity of median SNAPs were significantly different between normal and CTS groups $(\mathrm{p}<0.001)$. Although patients with milder involvement had similar distal latency to controls ( $\mathrm{p}=0.977$ ), SNAP amplitude and conduction velocity, being still in the normal ranges, were significantly different from controls $(\mathrm{p}<0.001)$. SNAP wrist to palm amplitude ratio was $0.81 \pm 0.12$ in controls. In 87 hands mean amplitude ratio was significantly lower than it was in normal group $(0.53 \pm 0.23$, p $<0.001)$ but the remaining forty hands with milder involvement had a mean amplitude ratio $(0.75 \pm 0.23)$ similar to controls $(\mathrm{p}=0.127)$. There was a significant reduction of SNAP amplitudes at the wrist compared to the palm in $54 \%$ of hands. Comparison of amplitudes between wrist and palm segment may give information about conduction block.
\end{abstract}

Key words: Carpal tunnel syndrome, Conduction block, Segmental conduction velocity.

(This article was submitted Feb. 28, 2000, and was accepted Oct. 20, 2000)

\section{INTRODUCTION}

Carpal tunnel syndrome (CTS) is the most common entrapment neuropathy in clinical practice. In compressive neuropathies there is both axonal degeneration and conduction block. In CTS, conduction block can be confirmed by comparing the SNAP amplitudes proximal and distal to the lesion. Although there is ample literature compassing electrophysiological features of CTS, how the compression affects the distal segment of the nerve below the compression is reported in few studies. We investigated sensory nerve potentials from the median nerve at the wrist and palm to determine the conduction block and changes at the distal segment of the median nerve in mild CTS patients.

\section{MATERIALS AND METHODS}

Control values were obtained from 61 hands of 48 healthy persons. We studied 189 hands of 156 consecutive patients diagnosed or suspected of CTS during a two-year period. Those subjects having severe conduction slowing (distal motor latency $>4.4$ ms, absent SNAP) were not included in the study.

All studies were performed by the same investigator with a Nihon Kohden 4-channel EMG machine. The filter band-pass was $2 \mathrm{~Hz}$ to $3 \mathrm{kHz}$ 
for motor studies and $20 \mathrm{~Hz}$ to $3 \mathrm{kHz}$ for sensory studies. The compound muscle action potential was recorded with surface electrodes from the abductor pollicis brevis muscle. The median nerve was stimulated $2 \mathrm{~cm}$ proximal to the distal wrist crease by a hand-held stimulator with $2 \mathrm{~cm}$ inter-electrode distance at the wrist. Stimulus duration was $0.2 \mathrm{~ms}$, sweep speed was $2 \mathrm{~ms} /$ division and amplitude gain was $5 \mathrm{mV}$. Measurements were done by a tape measure. Sensory nerve action potentials (SNAP) were obtained antidromically and were recorded by ring electrodes placed at the proximal and distal interphalangeal joints of the first and second digits. The palm stimulation site of the median nerve was 4 $\mathrm{cm}$ distal to the wrist crease on the line connecting the wrist and web space between the index and middle finger. Sweep speed was $2 \mathrm{~ms} /$ division and gain was $10 \mu \mathrm{V}$. Amplitudes of SNAP were measured from the isoelectric line to negative peak and distal latency from the onset point. Ulnar motor nerve conductions and concentric needle EMG investigations were also performed to exclude any additional or alternative disorders.

Statistical analysis was performed with SPSS for Windows 1997. Descriptive statistics for the mean, standard deviation, minimum and maximum values and two-tailed Student's t-test for comparative statistics were used.

\section{RESULTS}

The records of 127 symptomatic hands in 109 patients were included in this study. There were 10 males $(20.8 \%)$ and 38 females $(79.2 \%)$ aged between $17-80$ years (mean $40.5 \pm 10.4$ years) in the control group, and 22 males (20.2\%), 87 females (79.8\%) aged between 23-82 years (mean $43.5 \pm$ 11 ) in the CTS group. There were no significant differences in age and sex distribution between groups ( $\mathrm{p}=0.121$ and $\mathrm{p}=0.926$ respectively).
Mean values of electrophysiological parameters in normal and CTS groups are given in Table 1. The latency, amplitude and velocity of median SNAPs were significantly different between the normal and CTS groups $(\mathrm{p}<0.001)$. Eighty-seven $(68 \%)$ symptomatic hands showed slowed median nerve conduction velocity in thumb (MI) and index (MII) fingers; and in this group (PGI) all conduction parameters were significantly different from controls $(\mathrm{p}<0.001)$. The remaining forty hands (PGII) had either MI or MII or segmental wrist-palm (PW) slowing. The PGII group had similar distal latency with controls $(\mathrm{p}=0.977)$, but SNAP amplitude and conduction velocity, while within normal ranges, were significantly different from controls $(p<0.001)$. Twelve hands revealed only segmentary slowing. Although MI and MII distal latency and velocities were in the normal range, they were still slower than those of controls $(p<0.001)$.

The mean values of SNAP amplitudes after palm and wrist stimulation are shown in Table 2. SNAP wrist to palm amplitude ratio was $0.81 \pm 0.12$ (the lowest value 0.6 ) in controls. PGI showed a significantly lower mean amplitude ratio than the normal group $(0.53 \pm 0.23, \mathrm{p}<0.001)$. In the remaining forty hands with milder involvement (PGII) the mean amplitude ratio was $0.75 \pm 0.23$ which was not different from controls $(\mathrm{p}=0.127)$. Also, it was similar to that found in hands with only MI, MII or PWH slowing. In the first group 60 $(69 \%)$ of 87 hands and in the second, $9(22.5 \%)$ of 40 hands showed amplitude ratios less than the normal lower value. Only three of those having only segmentary slowing had less than normal amplitude ratios.

When palmar SNAP amplitude was correlated with wrist to palm amplitude ratio, to investigate whether there was a relationship between axonal involvement and focal demyelination, no significant correlation was observed $(\mathrm{r}=0.056, \mathrm{p}=0.607)$.

Table 1. Sensory conduction values in normals and CTS

\begin{tabular}{lcccccc}
\hline \multicolumn{3}{c}{ Normal group } & \multicolumn{3}{c}{ CTS group } \\
& $\begin{array}{c}\text { Latency }(\mathrm{ms}) \\
\text { Mean } \pm \text { SD }\end{array}$ & $\begin{array}{c}\text { Amplitude }(\mathrm{mV}) \\
\text { Mean } \pm \text { SD }\end{array}$ & $\begin{array}{c}\text { Velocity }(\mathrm{m} / \mathrm{s}) \\
\text { Mean } \pm \text { SD }\end{array}$ & $\begin{array}{c}\text { Latency }(\mathrm{ms}) \\
\text { Mean } \pm \text { SD }\end{array}$ & $\begin{array}{c}\text { Amplitude }(\mathrm{mV}) \\
\text { Mean } \pm \text { SD }\end{array}$ & $\begin{array}{c}\text { Velocity }(\mathrm{m} / \mathrm{s}) \\
\text { Mean } \pm \text { SD }\end{array}$ \\
\hline MII & $2.5 \pm 0.3$ & $36.5 \pm 13.5$ & $56.1 \pm 5.9$ & $3.5 \pm 0.4^{*}$ & $17.7 \pm 9.6^{*}$ & $40.3 \pm 5.4^{*}$ \\
MP & $1.3 \pm 0.2$ & $43.7 \pm 15.8$ & $57.2 \pm 6.1$ & $1.4 \pm 0.2^{*}$ & $25.7 \pm 12.0^{*}$ & $50.8 \pm 4.7^{*}$ \\
PW & & & $53.6 \pm 5.7$ & & $35.2 \pm 5.2^{*}$ &
\end{tabular}

MII, antidromic sensory conduction values from wrist to digit2; MP, antidromic sensory conduction values from palm to digit2; PW, wrist to palm segment conduction velocity. ${ }^{*} \mathrm{CTS}$ versus normal, $\mathrm{p}<0.001$. 
Table 2. Comparison of palm and wrist amplitudes between normals and patient groups

\begin{tabular}{lcc}
\hline & Palm $($ Mean \pm SD) & Wrist $($ Mean \pm SD) \\
\hline Normal & $43.7 \pm 15.8$ & $36.5 \pm 13.5$ \\
PGI & $24.4 \pm 10.8$ & $15.7 \pm 6.7$ \\
PGII & $28.7 \pm 13.8$ & $22.2 \pm 13.0$ \\
\hline
\end{tabular}

\section{DISCUSSION}

Carpal tunnel syndrome is the most common and widely investigated entrapment neuropathy. In most previous studies, all interest was focused on the carpal tunnel segment. In compressive neuropathies, comparison of amplitudes proximal and distal to the lesion can distinguish demyelination from axonal degeneration. There are few studies evaluating this in CTS.

Buchthal and Rosenfalck ${ }^{1)}$ reported higher SNAP amplitudes, still lower than normal, at the palm compared to the wrist in 9 of 14 CTS hands with near-nerve recording. Kimura ${ }^{2}$ found significantly larger compound muscular action potential amplitude (CMAP), but not SNAP amplitude at the palm compared to the wrist. According to Lesser et al. a greater than $20 \%$ CMAP and greater than $60 \%$ SNAP amplitude reduction were indicative of focal demyelination $^{3)}$. They found that in $39 \%$ of hands CMAP palm/wrist amplitude ratios exceeded the upper limit of normal, and in 36\% SNAP palm/wrist amplitude ratios were greater than the upper limit of normal. Guglielmo et al., evaluating a large CTS group, suggested that to infer conduction block a greater than 50\% reduction in SNAP amplitude and a greater than $30 \%$ reduction in CMAP amplitude is required $^{4)}$. Johnson reported that a SNAP amplitude reduction greater than $60 \%$ suggests neuropraxia ${ }^{5}$, but there is no actual data supporting this indication.

Stimulation of the median nerve at the palm may activate the distal segments of the nerve near the motor point. This may result in a greater latency difference between the palm and wrist, and segmental slowing ${ }^{6}$. In order to avoid fallacious results we only evaluated sensory fibers. In our study, according to values in 61 asymptomatic hands a greater than $40 \%$ reduction in SNAP amplitude is required to infer conduction block. Considering all CTS hands, $36 \%$ of hands had significantly reduced SNAP amplitudes at the wrist compared to the palm. Ten hands (8\%) had no amplitude drop across the wrist segment.
SNAP amplitude is the best indicator of large diameter sensory axonal health and the amplitude drop indicates axonal loss. Considering all hands, SNAP amplitudes after palmar stimulation were significantly reduced compared to controls. This difference indicates a quota of axonal degeneration.

In two of twelve hands with normal PW velocity and three of eighteen hands with only MI slowing, amplitude loss was greater than $40 \%$. This suggests that axonal loss might be an early feature in CTS. But the comparison groups were very small, and in all hands segmentary velocities were also slow indicating conduction block. In $31 \%$ of more significantly involved hands no significant amplitude loss was recorded. These heterogeneous findings make it difficult to say whether axon loss or demyelination begins first. However, there was no correlation between palm SNAP amplitudes and the respective SNAP wrist to palm ratios suggesting that focal demyelination is not related to axonal involvement.

\section{CONCLUSION}

Distinguishing axonal involvement from conduction block might be useful in deciding which treatment to apply and predicting prognosis in CTS. Comparing SNAP amplitudes between wrist and palm segments may give information about conduction block.

\section{REFERENCES}

1) Buchthal F and Rosenfalc A: Sensory conduction from digit to palm and from palm to wrist in carpal tunnel syndrome. 1971, J Neurol Neurosurg Psychiatry, 34: 243-252.

2) Kimura J: The carpal tunnel syndrome: Localization of conduction abnormalities within the distal segment of the median nerve. 1979, Brain, 102: 619-635.

3) Lesser EA, Venkatesh S, Preston DC, et al.: Stimulation distal to the lesion in patients with carpal tunnel syndrome. 1995, Muscle Nerve 18: 503-507.

4) Guglielmo GD, Torrieri F, Repaci M, et al.: Conduction block and segmental velocities in carpal tunnel syndrome. 1997, Electroencephalogr Clin Neurophysiol, 105: 321-327.

5) Johnson EW: Should immediate surgery be done for carpal tunnel syndrome? 1995, Muscle Nerve, 18: 658-659.

6) Kimura J: Facts, fallacies and fancies of nerve stimulation techniques. In: Electrodiagnosis in Disease of Nerve and Muscle: Principles and Practice, 2nd ed. 1989, Philadelphia: FA Davis Company, pp 139-166. 